\title{
Fatigue of automotive engine cylinder heads - A new model based on crack propagation
}

\author{
Mathieu Béranger ${ }^{1, *}$, Guillaume Morin ${ }^{1}$, Sarah Saanouni ${ }^{2}$, Alain Koster $^{2}$, and Vincent Maurel $^{2}$ \\ ${ }^{1}$ Direction de la Mécanique, RENAULT, 78084 Guyancourt cedex, France \\ ${ }^{2}$ MINES ParisTech, PSL Research University, MAT - Centre des Matériaux, CNRS UMR 7633, BP 8791003 Evry, France
}

\begin{abstract}
The main reliability issue encountered during cylinder head development is the appearance of fatigue cracks during severe endurance tests. Some of these cracks can initiate in the coolant water jacket and lead to the complete part failure. They are mainly associated with the combination of combustion pressure alternate stresses and high cycle fatigue load in infinite life domain. However, Haigh or Dang Van analysis issued from 3D Finite Element Analysis reveals several issues: crack initiations are not correctly located and scatter of crack sizes are not predicted. To overcome these difficulties, a new fatigue model dedicated to cylinder heads in Aluminum - Silicon alloy has been developed in Renault powertrain division. The model is based on fatigue crack growth modelling in order to take into account various and combined load ratio in one hand, and typical characteristics of the microstructure of casting alloy in the other hand. This paper presents the theoretical basis of this model and the associated identification methodology for two different aluminum alloys.
\end{abstract}

\section{Cylinder Head background}

Design of modern automotive internal combustion engines has become an increasing challenging task in the past years due to increasing constraints in terms of performance, fuel economy and emissions.

Mechanical and thermal loads increase on high specific power output engines, mainly turbocharged direct injection Diesel and gasoline engines, has made more and more difficult to reach reliability targets. In parallel, development costs reduction calls for an increase in simulation model accuracy in order to get directly the optimized design with reduced physical validation tests.

\subsection{Fatigue failure mode}

Cylinder head is a critical and complex part in an internal combustion engine. It has a great impact on performance via intake and exhaust aerodynamic and it undergoes high mechanical and thermal loads directly linked to combustion process and design of the part.

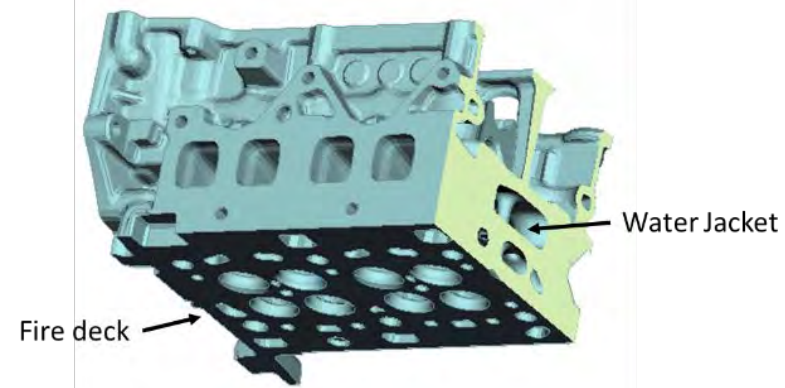

Fig. 1. Cylinder head cut (Diesel engine)
The main reliability issue encountered during cylinder head development is the appearance of fatigue cracks during severe endurance tests.

These cracks can initiate in the coolant water jacket and can lead to the complete part failure.

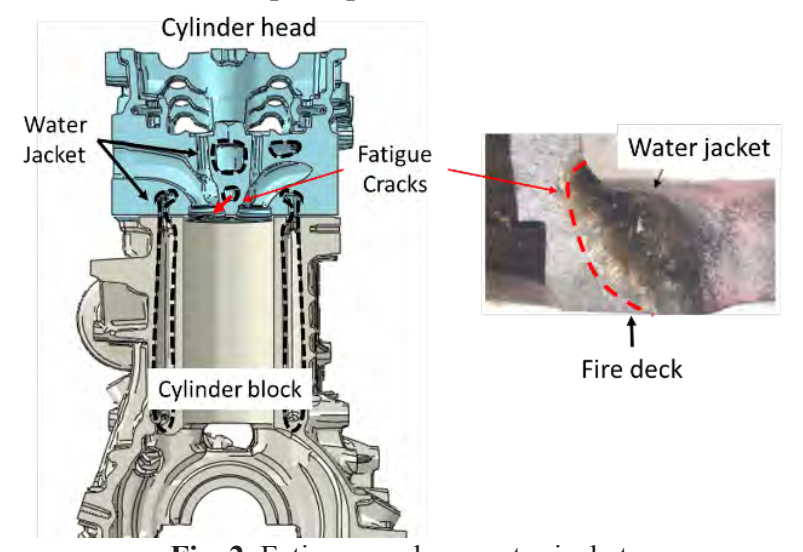

Fig. 2. Fatigue crack on water jacket

\subsection{Water jacket loading}

Cracks initiated in the coolant water jacket are associated mainly with combustion pressure alternate stresses and high cycle fatigue (HCF).

However, in the next discussion we will consider several kinds of loadings:

- Static loading due to tightening on cylinder block, interference fitting of seats;

- Thermal loading due to gas combustion flow;

* Corresponding author: mathieu.beranger@,renault.com 
Because of the engine power variation, that loading is cyclic and therefore creates thermo-mechanical stresses.

- Pressure loading at each combustion in the cylinder

The range and frequency of both thermal and pressure cyclic loadings are not the same. Actually, thermal loading will induce about $10^{4}$ cycles of low cycle fatigue (LCF) range (typically with a load ratio $\mathrm{R}=0.1$ ) while pressure loading induces about $10^{7}$ cycles of high cycle fatigue (HCF) range (typically with a load ratio $\mathrm{R}=0.7$ ). Figure 3 illustrates the decomposition of the different loading during an engine elementary test bench.

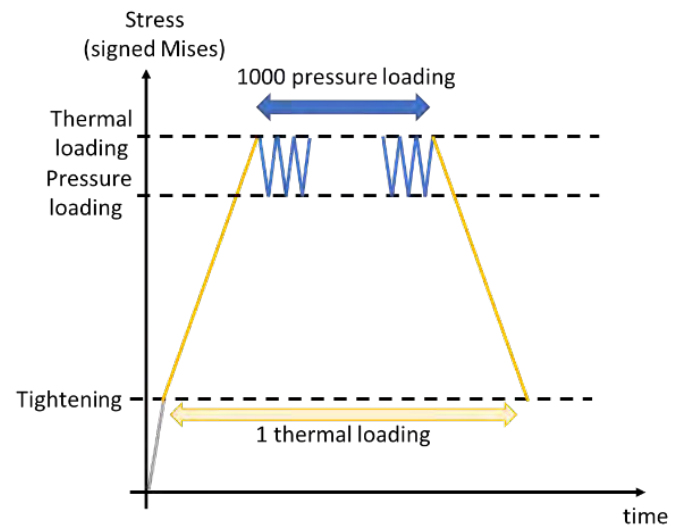

Fig. 3. Decomposition of loading on water jacket

The quantity of interest is the ratio of the number of pressure loadings added on water jacket for each thermal loading, e.g. 1000 in Fig. 3. This ratio depends on the type of engine test bench, in particular the engine speed applied during the test.

\subsection{Material characteristic}

Aluminum - Silicon alloys are currently used for cylinder head for decades to reduce weight and therefore increase fuel efficiency. Material casting (in our case gravity casting) are known to generate coarse microstructure and shrinkage cavities associated to the dendritic structure of these alloys. In our case, the size of typical shrinkage cavities is ranging from $0.1 \mathrm{~mm}$ to $0.5 \mathrm{~mm}$.
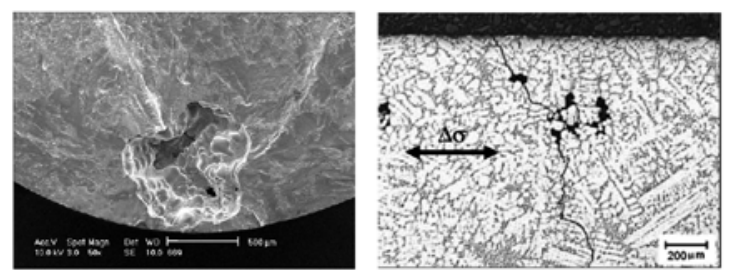

Fig. 4. Crack initiation on shrinkage cavity and subsequent crack propagation $[1,2]$

For both HCF [3, 4] and LCF loadings [5], this kind of defects can be considered as the main sites of crack initiation. After initiation, cracks firstly develop in the so-called short crack range and can propagate to reach long crack domain [6].

This study will cover the analysis of two kinds of aluminum-silicon alloy: the first one is a $3 \%$ copper alloy (historical choice of Renault cylinder head) and the second one is a new $0.5 \%$ copper alloy developed for high performance engines.

\section{Crack propagation model for water jacket}

\subsection{Historical context}

Tests bench performed on 2L Diesel engine have shown the role of both thermal and combustion loading on water jacket damage.

Measurements of crack lengths have been achieved for different test benches associated to different thermalmechanical fatigue loadings, Fig. 5:

- $\quad P_{\max }$ tests: engine is maintained at maximum power. This test induces only pressure loading stresses.

- Hot-Cold tests: both thermal and pressure loadings are applied on cylinder head.

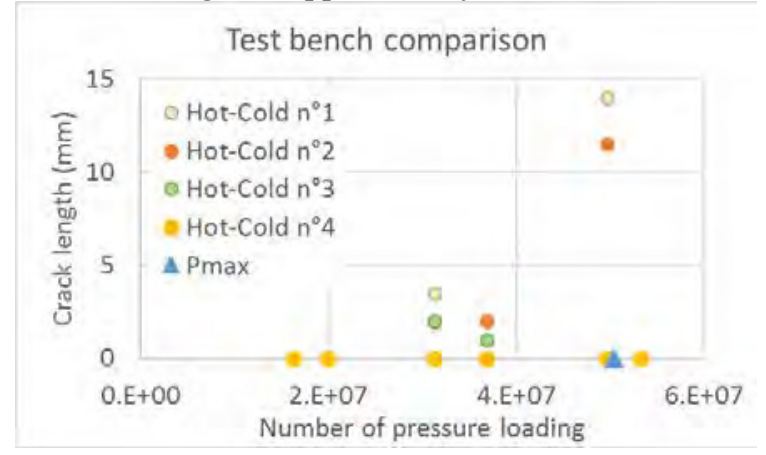

Fig. 5. Thermal-Mechanical loading effect on crack length for bench $P_{\max }$ and Hot Cold tests

While many cracks are observed at the end of Hot-Cold tests, for higher number of pressure loading, $P_{\max }$ test has not revealed any crack on water jacket.

To prevent appearance of cracks, classical methodologies use stresses estimated from 3D Finite Element Analysis to determine the level of a fatigue safety factor based on Dang Van criterion [7] as illustrated in Fig. 6. In this case, the stress path is represented in a mesoscopic shear $\tau(\mathrm{t})$ versus mesoscopic hydrostatic stress $\sigma_{\mathrm{H}}(\mathrm{t})$ diagram.

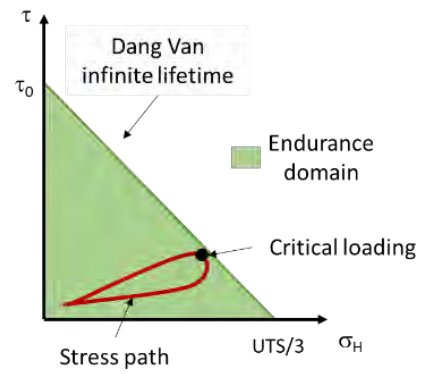

Fig. 6. Illustration of the Dang Van criterion The criterion is defined by the boundary between infinite life (endurance domain) and fatigue. Component life for a load path contained completely below the Dang Van 
Criteria line will be infinite. However, this approach does not allow to consider simultaneously low and high cycles fatigue.

Another classical methodology to take into consideration variable amplitude stresses, is to consider damage accumulation.

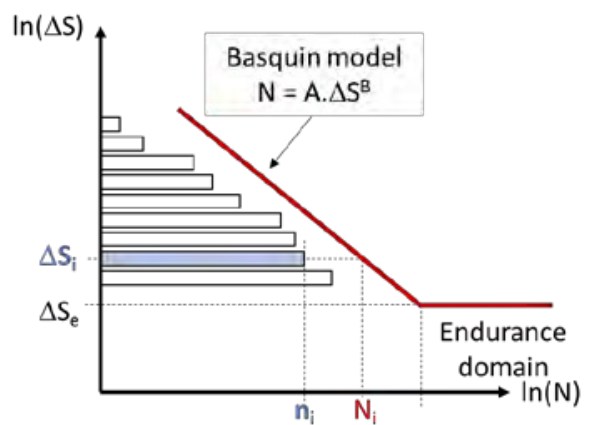

Fig. 7. Damage calculation on S-N curve

Using the linear damage rule of Miner - Palmgreen, it is possible to estimate, for various loading the total damage $D$ :

$$
D=\sum_{i} \frac{n_{i}}{N_{i}}
$$

Classically, High Cycle Fatigue and Low Cycle Fatigue are implemented separately.

\subsection{Crack propagation model}

To overcome these difficulties, a new fatigue model, based on fatigue crack growth modelling, has been developed in Renault powertrain division.

This approach has two main advantages:

- It takes into account typical characteristics of microstructure of casting alloy. Distribution of size and localisation of porosities allow performing probabilistic analysis of fatigue lifetime, and can therefore explain observed experimental scatter.

- Effect of various and combined loading is used to evaluate fatigue lifetime.

The model formulation is defined by:

$$
\frac{d a}{d N}=\frac{d a}{d N}\left(\Delta K_{L C}\right)+\frac{N_{S C}}{N_{L C}} \times \frac{d a}{d N}\left(\Delta K_{S C}\right)
$$

With the following notations:

- $\Delta K_{L C}$ is the amplitude of stress intensity factor for cycles of large amplitude, such as thermal loading ;

- $\frac{d a}{d N}\left(\Delta K_{L C}\right)$ is the crack growth rate associated to large cycles;

- $\Delta K_{S C}$ is the amplitude of stress intensity factor for cycles of small amplitude, such as pressure loading;
- $\frac{d a}{d N}\left(\Delta K_{S C}\right)$ is the crack growth rate associated to large cycles;

- $\frac{N_{S C}}{N_{L C}}$ is the ratio of numbers of small cycles and large cycles

Initial crack growth rate of water jacket is then estimated based on a typical flaw size of $300 \mu \mathrm{m}$.

Next paragraph details this model, and describes the global methodology applied to identify those parameters: crack propagation tests, mathematical formulation and correlation with variable stress loadings.

\section{Variable amplitude crack growth methodology}

The design of the crack propagation model, developed in equation (2), is divided into four steps, described hereafter:

- Estimation of Paris' law with threshold using Compact Tension tests,

- Validation of long crack propagation model on small cracks using notched specimen,

- Crack propagation tests with variable amplitude loadings,

- Correlation with engine tests.

The following nomenclature will be used in the next sections

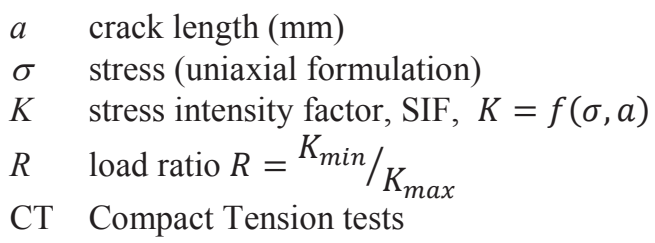

\subsection{Long crack propagation model}

The determination of propagation model is based on the determination of the coefficients of the Paris law:

$$
\frac{d a}{d N}=C . \Delta K^{m}
$$

Where

- $\quad \mathrm{m}$ and $\mathrm{C}$ are two material dependent parameters,

- $\Delta K$ the amplitude of stress intensity factor

- $\quad K=\sigma \cdot F I(a) \cdot \sqrt{\pi a}$

with FI the shape function associated to the specimen shape

CT tests, according to ASTM standard [8] have been achieved for different load ratios $\mathrm{R}$ ranging from 0.1 to 0.7 , at $150{ }^{\circ} \mathrm{C}$, which is the average temperature of in use water jacket. Those tests are currently used to characterize FCGR for long cracks. Crack length measurements is achieved using continuous potential 
drop technique calibrated on optical inspection of crack length.

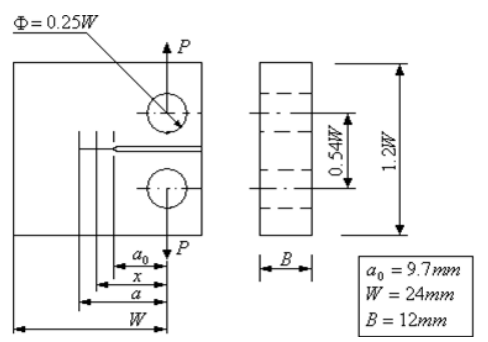

Fig. 8. CT sample definition

The next figure presents for all CT tests the evolution of fatigue crack growth rate (FCGR) vs the amplitude of stress intensity factor, these tests corresponds to the $0.5 \%$ copper aluminum alloy.

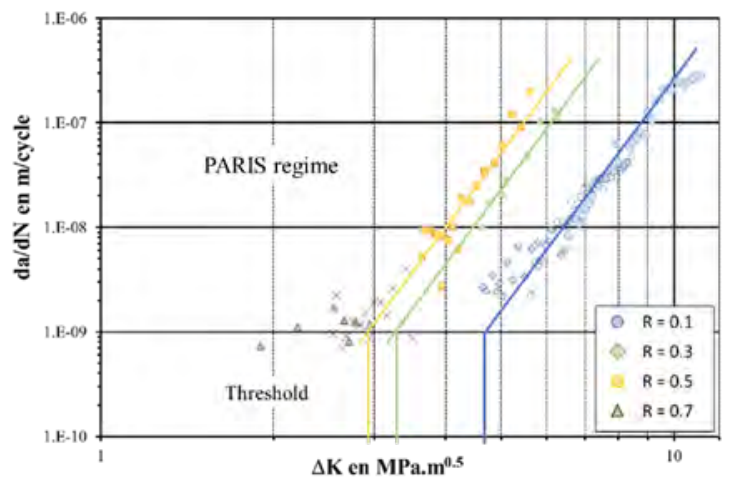

Fig. 9. CT tests results for $0.5 \%$ copper aluminum alloy CT tests show an obvious dependency of the threshold $\Delta K_{t}$ as a function of the load ratio $R$, Fig. 9. According to Elber [9], such a dependency can be corrected by taking into account only a part of the SIF amplitude, considering the SIF value needed to open the crack $K_{\text {open }}$. Thus, the effective stress intensity factor $\Delta K_{\text {eff }}$ is defined as:

$$
\Delta K_{\text {eff }}=K_{\text {max }}-K_{\text {open }}
$$

and the crack propagation model by:

$$
\frac{d a}{d N}=C . \Delta K_{e f f}^{m}
$$

with $C$ and $\mathrm{m}$ material parameters being independent of $R$ load ratio.

To determine the $K_{\text {open }}$ value as a function of $R$, we first assume that for $R \geq 0.7$, the crack remains opened during the whole fatigue cycle. For other load ratio $R, K_{\text {open }}$ is obtained as the gap between the asymptotic threshold value and the linear interpolation of experimental $\Delta K_{t}$, Fig. 10. It is thus assumed that for SIF values lower than $K_{\text {open }}$, cracks will not propagate.

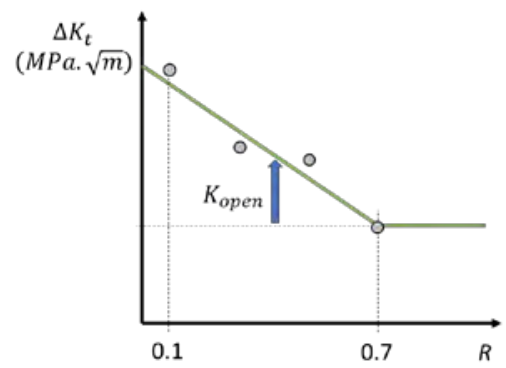

Fig. 10. $K_{\text {open }}$ correction

This model yields to a master curve describing any tested $R$ ratio leading to a fairly good accuracy for both aluminum alloys with $3 \%$ and $0.5 \%$ copper, respectively Fig11 and 12.

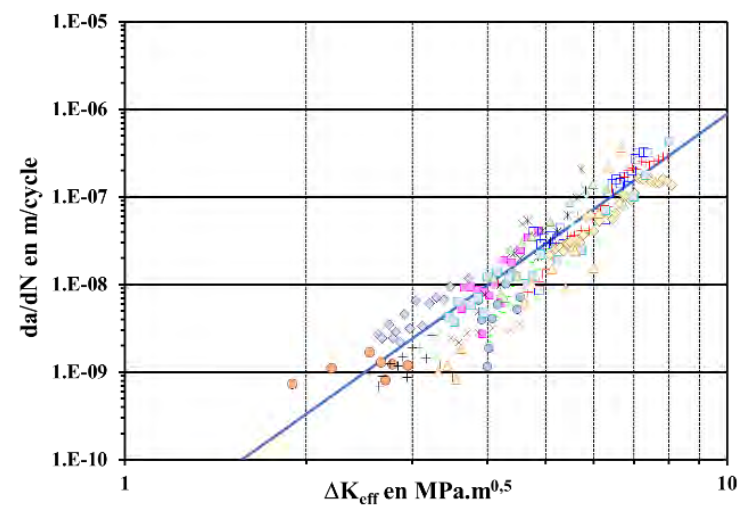

Fig. 11. Long crack growth rate model (3\% copper alloy)

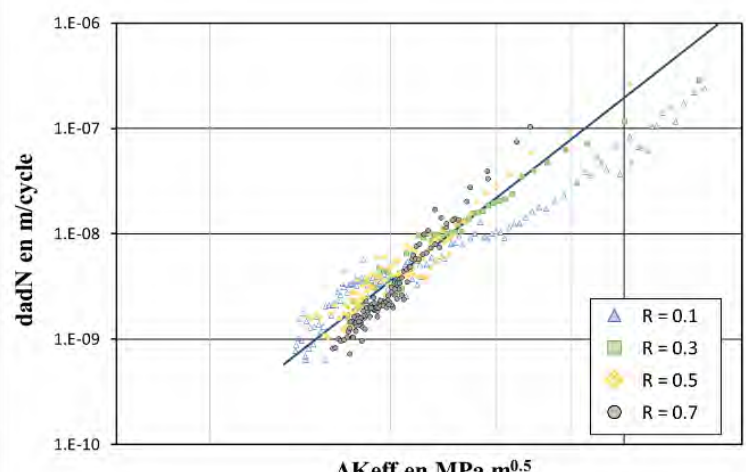

Fig. 12. Long crack growth rate model ( $0.5 \%$ copper alloy) It is worth noting that observed scatter, is the direct consequence of $\mathrm{CT}$ tests results for highest applied $\Delta K$ values. However, this study aims at focusing on intermediate $\Delta \mathrm{K}$ values where the model is very consistent.

\subsection{Small crack propagation validation}

Crack propagation mechanisms are not similar for short and long crack [6]. As our objective is to take into account initiation of small crack on water jacket, it is important to test the proposed crack propagation model issued from long cracks tests onto small cracks propagation ones.

Special geometry sample has been developed for that purpose [4] using notched sample with rectangular section. The initial notch is a semi-elliptical cut of about $500 \mu \mathrm{m}$ in width achieved by electro-discharge machining (EDM) on the largest flat face of the gage area.
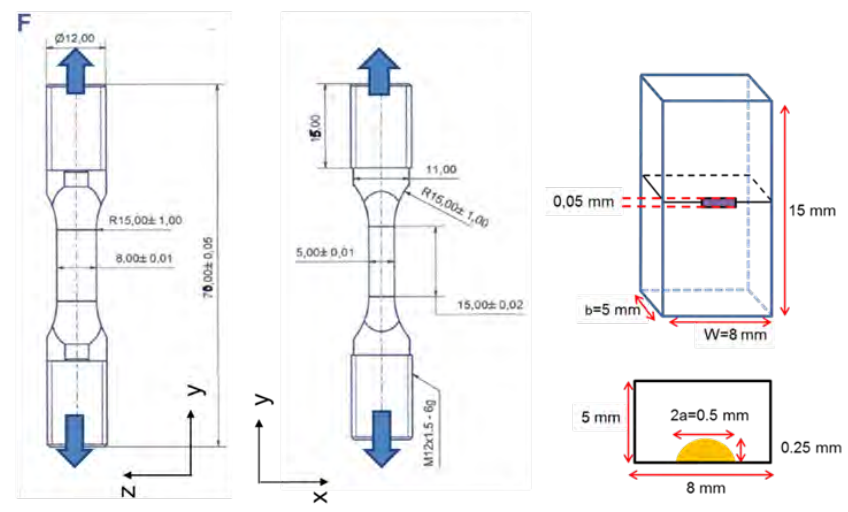
Fig. 13. Sample definition for short cracks study

Unfortunately, due to both the small size of the gage area and of the notch, this geometry prevent from the use of potential drop technique for crack length tracking.

Therefore, crack length evolution is derived from in situ image analysis using an optical microscope.

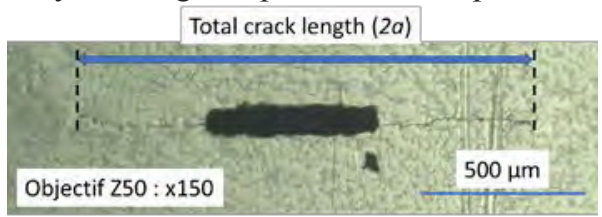

Fig. 14. Crack growth image analysis

Tests have been driven at $150{ }^{\circ} \mathrm{C}$, for different load ratio ranging from -1 . to 0.7 .

The following picture represents the crack growth rate $\frac{d a}{d N}$ as a function of the effective stress intensity factor $\Delta K_{\text {eff }}$. It is worth noting that $\Delta K_{\text {eff }}$ takes into consideration the $K_{\text {open }}$ correction yielded by equation (5).

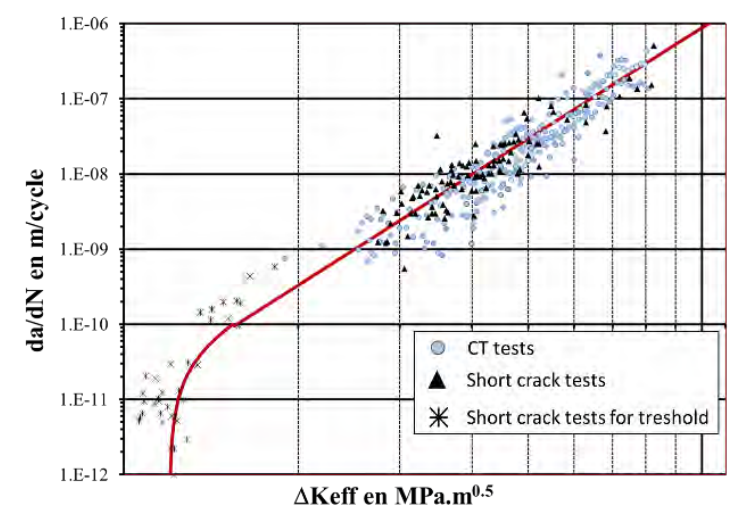

Fig. 15. Short crack growth rate model (3\% copper alloy) First analysis of those results confirms the consistency of the long crack model for cracks initiated on a small notch.

However, for low $\Delta K_{e f f}$ values, significant increase of FCGR is observed as compared to the unified Paris model. This locally high FCGR is consistent with the influence of short crack on FCGR [6].

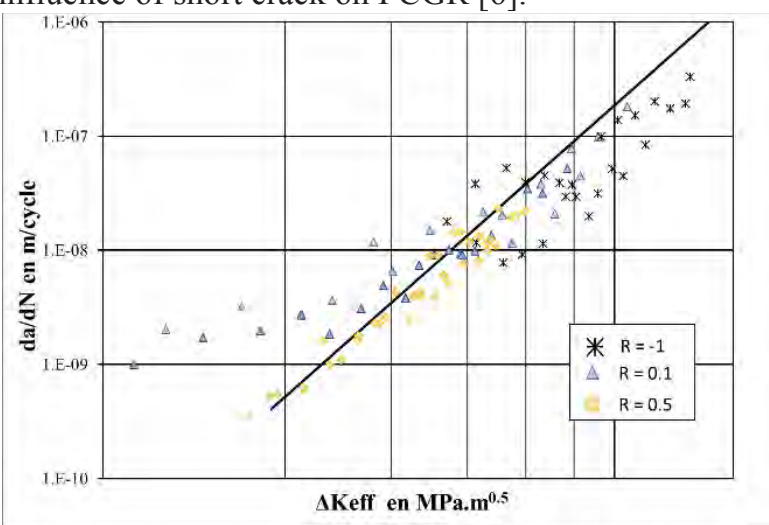

Fig. 16. Short crack growth rate model ( $0.5 \%$ copper alloy) Those results and their impact on global methodology are still under analysis for the new aluminum alloy.

\subsection{Crack propagation with variable amplitude loading}

As discussed in the section devoted to the context of this study, water jacket of cylinder head bears complex loadings: first, the tightening of the cylinder head on peripheric parts, which is kind of a permanent static load, and then 2 dynamic loadings: thermal one and cylinder pressures.

To get as close as possible to real loads conditions, specific propagation tests have been carried out. Based on the same geometry of small cracks tests, we proposed to combine low amplitude and high frequency cycles referred to "small cycles" (corresponding to cylinder pressure cycles), on high amplitude and low frequency cycles, designated by "large cycles" (corresponding to the starting and stopping of the engine).

Those tests are defined with 3 parameters: the ratio between the respective number of small and large cycles (from 500 to 1000 in our case), the load ratio for small and large cycles ( 0.7 and 0.1 in our application).

The next figure shows the diagram load program test.

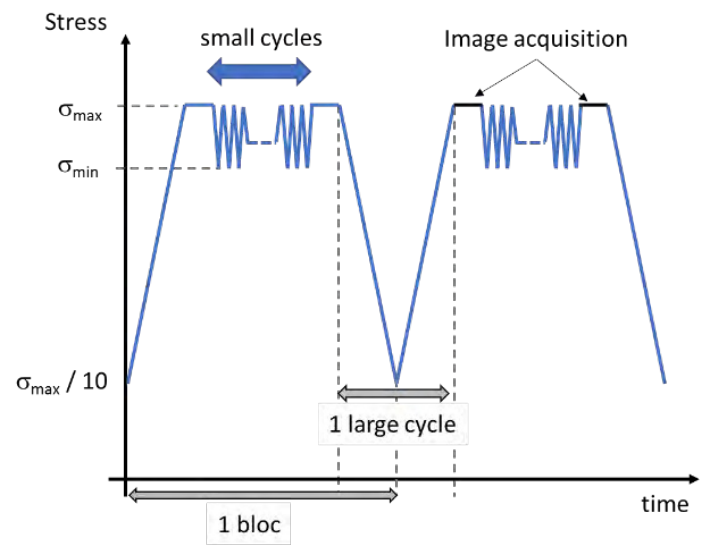

Fig. 17. Crack tests with combined loading The intent of the study is to understand the effect of combination of short cycles and long cycles on FCGR, thus we proposed a first simple analysis of tests results.

Only 3\% copper alloy results will be discussed in the next paragraph as recent tests for $0.5 \%$ copper alloy are still in progress and need additional analysis.

Firstly, we consider only the effect of small cycles to evaluate the FCGR, which assumption yields to:

$$
d a / d N \approx \Delta a / \Delta N_{S C}
$$

where $\Delta N_{S C}$ is the number of small cycles measured during the increment of $\operatorname{crack} \Delta a$.

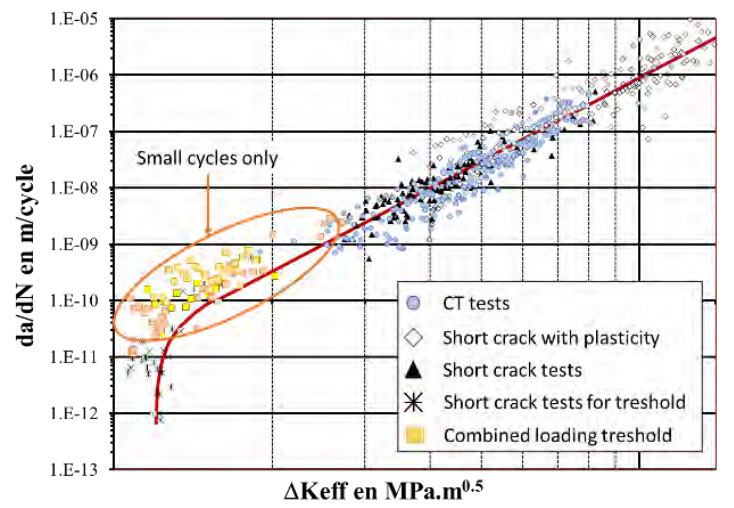

Fig. 18. Comparison of small cycles effect with Paris model for long cracks ( $3 \%$ copper aluminium alloy) 
This assumption, leads to an overestimation of the FCGR with respect to the master curve, Fig. 18. Moreover, whereas these tests have been achieved using notched specimens, no evidence of short crack behavior is observed.

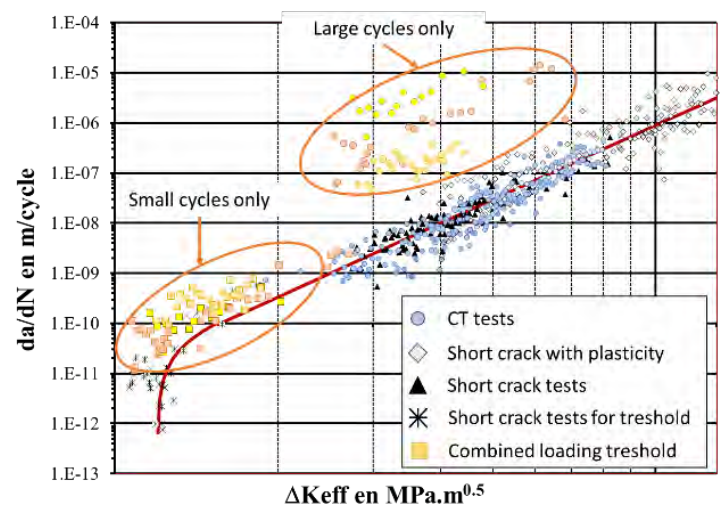

Fig. 19. Comparison of large cycles effect with Paris model for long cracks ( $3 \%$ copper aluminium alloy)

Secondly, we consider only the effect of large cycles to evaluate the FCGR, which assumption yields to:

$$
d a / d N \approx \Delta a / \Delta N_{L C}
$$

where $\Delta N_{L C}$ is the number of large cycles measured during the increment of crack $\Delta a$. In this case, FCGR is ten times higher than the master curve.

As a partial conclusion, variable amplitude loading shows a dependency of crack growth on both large and small cycles.

\subsection{First interpretation and limits}

The first interpretation is to separate, with a cumulative damage model, the effect of large and small cycles.

For large cycles, an incremental approach is applied to estimate, for a constant step of crack length ( $d a=$ $50 \mu \mathrm{m})$ :

- on one hand, the number of large cycles $d N_{k}$ performed during the tests with respect to the following picture where one block is equalled to one large cycle.

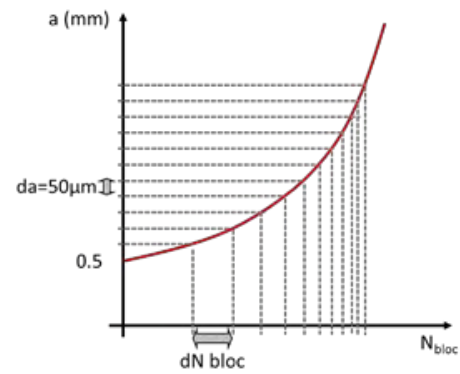

Fig. 20. Estimation of realised $d N_{k}$ large cycles

- on the other hand, the level of $\Delta K_{\text {eff }}$ given by equations (4) and (5). The considered SIF values are limited to large cycles (here $R=0.1$ ) and are associated to a given $d N_{-} P_{k}$ according to the Paris' model (6).

Finally, the increment of damage associated to crack growth due to large cycles is given by:

$$
d_{i}=\frac{\sum_{k=1}^{i} d N_{k}}{\sum_{k=1}^{i} d N_{-} P_{k}}
$$

For small cycles, the described methodology is slightly adapted. Considering only small cycles, the associated SIF values (here for $R=0.7$ ) are observed to be very close to SIF threshold derived from long crack analysis and the master curve. This is the reason why, we choose to extend the Paris law to low values of $\Delta K_{\text {eff. }}$.

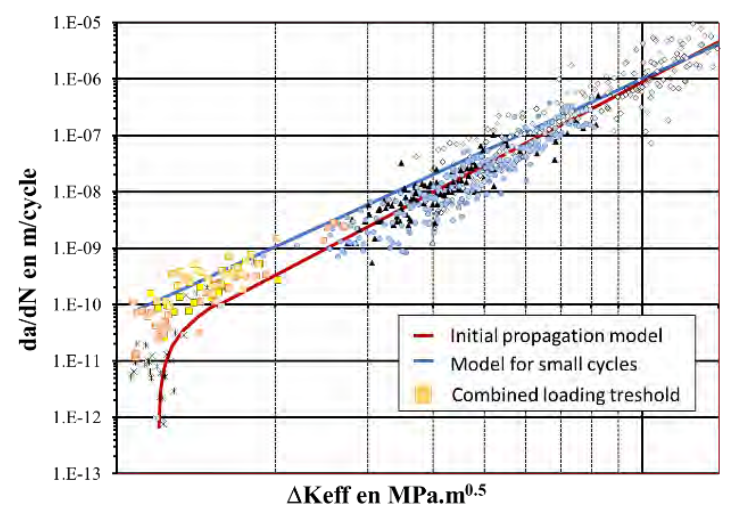

Fig. 21. Paris model extension for small cycles (3\% copper aluminium alloy)

This methodology is finally applied to combined loading testing analysing both the FCGR, Fig. 21, and, after integration of the Paris law, the crack length plot as a function of the number of cycles, Fig. 22.

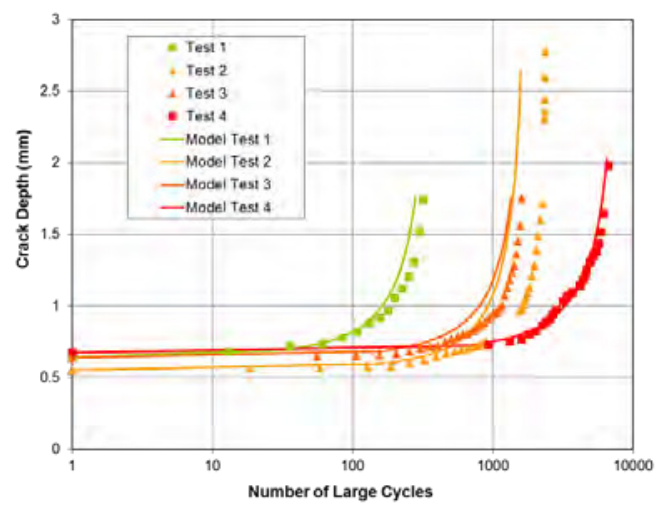

Fig. 22. Validation of crack growth model on combined loading ( $3 \%$ copper aluminium alloy)

Finally, the chosen assumptions have led to a fairly good evaluation of the measurements, validating the interest of such a methodology, at least for that kind of aluminium alloy.

\subsection{Initial size of water jacket's flaw}

Once the proposed model (2) has been fitted on different kinds of tests, it is necessary to define the initial size of critical flaw leading to crack initiation. This should be determined for the considered water jacket's.

First analysis is based on small cracks propagation tests. We have noticed that for machined elliptical notch of less than $300 \mu \mathrm{m}$, cracks started on other flaw induced by the process, initially present in the specimens. However, for an elliptical notch size of above $500 \mu \mathrm{m}$, cracks always iniate from the notch [4].

Another way to validate that initial size of the water jacket's flaw is based on S-N curve dispersion. Wöhler analysis allows us to define the dispersion of S-N curve. 
On the other hand, lifetime curve can be estimated with the crack propagation model (6) for different given size of initial flaw.

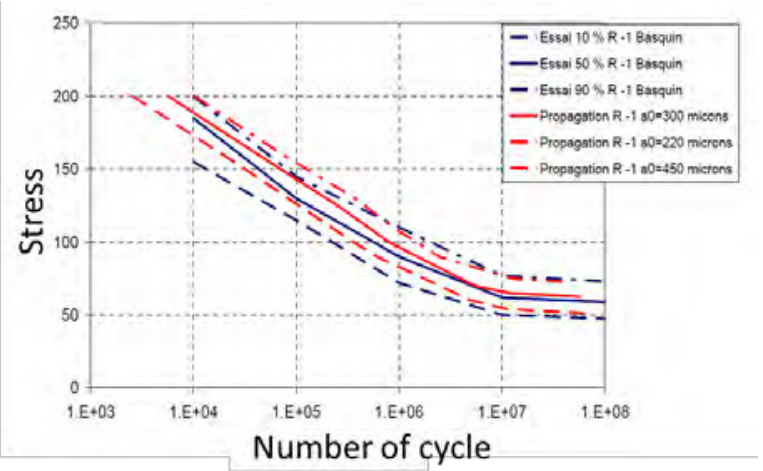

Fig. 23. Estimation of flaw size on S-N curve (3\% copper aluminium alloy)

As we can see in Fig. 23, a flaw size of $300 \mu \mathrm{m}$ is consistent with the average lifetime of S-N curve. This value of initial flaw will be used to estimate the initial FCGR for any location in the water jacket.

As a validation step, evaluation of cracks propagation has been achieved on the 2L Diesel engine for different kind of loadings.

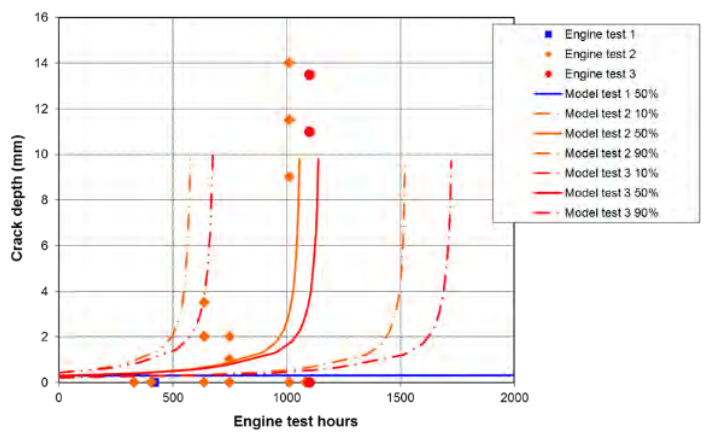

Fig. 24. Correlation of crack length on engine tests (3\% copper aluminium alloy)

For the Pmax test, the model confirms that the crack cannot propagate without thermal loading while a good correlation of crack length is noticed for Hot-Cold tests.

\section{Conclusions and perspectives}

The proposed methodology and criterion is used as a simulation standard for the cylinder head design for about 10 years.

The first interest is to improve the localization of critical area as compared to classical models based on crack initiation analysis. Actually, this criterion allows us to identify cracks due to thermal and pressure loadings where classical multi-axial criteria failed.

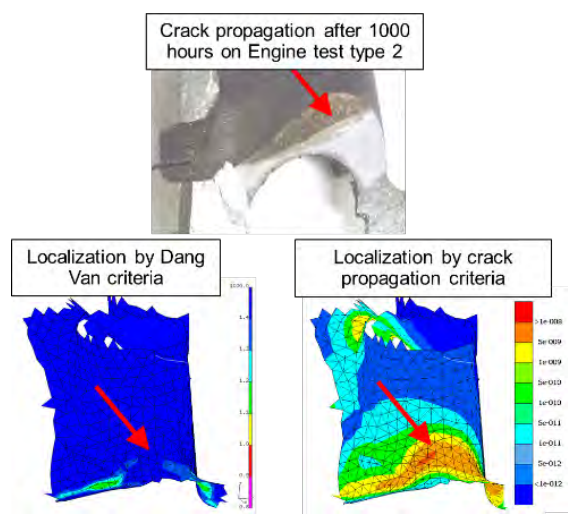

Fig. 25. Comparison of Dang Van criteria and dadN criteria on water jacket

Moreover, the proposed methodology is able to obtain a ranking of critical areas consistent with observed inservice damage, instead of classical initiation method leading to artefacts in damage localization and ranking. This kind of ranking is of first importance to focus on the main issues to solve for a reliable design of the cylinder head.

The same approach is currently under construction for a new aluminium silicon alloy with low copper content, for which first results have been presented in this study. Additional tests to understand deeper the interaction between large and small cycles will be discussed in a future work.

\section{References}

1. W.W. Bose-Filho, Al-Si cast alloys under isothermal and thermomechanical fatigue conditions, IJF (2007)

2. H. Mayer, Influence of cyclic loads below endurance limit or threshold stress intensity on fatigue damage in cast aluminium alloy 319-T7, IJF 27, p 129-141 (2005)

3. Shyam, et al. Scripta Materialia, 50(8) (2004), pp 1109-1114.

4. R. Salapete, $\mathrm{PhD}$ Thesis in French, Propagation de fissures dans les culasses Diesel (2007), Mines ParisTech

5. Dezecot, S., et al, Acta Materialia, 123 (2017), pp 24-34.

6. S. Suresh, R.O Richie, Propagation of short fatigue cracks, Int. Met. Rev. 29 (1984), pp 445-476

7. K. Dang Van, Advances in multiaxial fatigue, ASTM STP 1191 (1993), pp 120-130

8. Standard Test Method for Measurement of Fatigue Crack Growth Rates, ASTM E647 (2015)

9. W. Elber, The significance of fatigue cracks closure, ASTM STP, 486 (1971), pp 230-242 